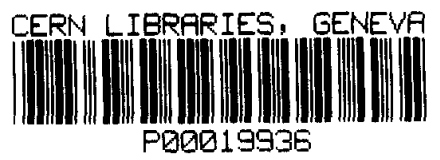

\title{
FAST LUMINOSITY MONITOR AT LEP
}

\author{
C.Bini, D.De Pedis, G.De Zorzi, G.Diambrini-Palazzi, G.Di Cosimo, \\ A.Di Domenico, P.Gauzzi, D.Zanello \\ Dipartimento di Fisica, Università "La Sapienza", Roma \\ $\&$ \\ INFN Sezione di Roma, Italy
}

\begin{abstract}
In 1990 the LEP-5 experiment measured luminosity at LEP by detecting the single bremsstrahlung photons emitted in the $\mathrm{e}^{+} \mathrm{e}^{-}$collisions. In 1991 the experiment was upgraded to exploit the intrinsic high speed of the method which allows to measure the luminosity of the single bunches of LEP. In this paper the LEP-5 upgrade is described and the results of a test performed are discussed.
\end{abstract}

(Submitted to Nuclear Instruments and Methods) 


\section{Introduction}

The measurement of luminosity at the Large Electron Positron collider (LEP) at CERN is essential in view of two different tasks: normalization of physics experimental data and machine performance optimization. The former requires a high degree of systematic accuracy on integrated luminosity, while the latter requires high statistics in order to get a quick evaluation of short term luminosity variations. Each LEP experiment is equipped with a luminosity monitor based on the detection of small angle Bhabha scattering [1]. Such monitors reach a typical systematic uncertainty below the $1 \%$ level, matching the required accuracy for LEP physics. However their relatively low event rate is not suitable for beam optimization purposes.

A fast luminosity monitor based on detection of single bremsstrahlung photons has been built and operated by the LEP-5 experiment. The results of a test experiment carried out in Interaction Point 1 (IP-1) of LEP in summer 1990, are described in Ref. $[2,3]$. A $1 \%$ statistical and $3-5 \%$ systematic uncertainty were obtained in 10 minutes runs.

During 1991 an upgrade of the LEP-5 experiment has been carried out with two objectives:

(a) to exploit the intrinsic high rate capability of the experimental method;

(b) to perform a luminosity measurement for each of the 4 bunches allowing bunch-to-bunch normalization. The latter can become crucial in case beam polarization will be implemented at LEP [4]:

Both objectives can be reached by utilizing a new read-out system.

In this paper, after having recalled the main features of the LEP-5 experiment (Sect.2), the new read-out system is described (Sect.3) and the results of a test performed in late 1991 are discussed (Sect.4).

\section{The LEP-5 experiment}

In this section the main features of the single bremsstrahlung method utilized by the LEP-5 experiment are summarized; more details can be found in Ref.[2].

Bremsstrahlung photons are emitted in the forward direction and travel along the LEP half straight section $(\approx 300 \mathrm{~m})$. Then they pass through a 2 (vertical) $x 5$ (horizontal) $\mathrm{cm}^{2}$ window in the vacuum pipe, just at the beginning of the 
arc, and reach the detector (see Fig.1). The LEP-5 detector consists essentially of a modular electromagnetic calorimeter made of lead and scintillating fibres capable to measure both energy and impact point position of the incident photons; its features are described in Ref.[5]. A low- $\mathrm{Z}$ absorber (2 radiation length of $\mathrm{LiH}$ ) is placed in front of the calorimeter in order to reduce the synchrotron radiation background to a negligible level.

At LEP the luminosity measurement, based on detection of single bremsstrahlung photons, has some features that must be stressed. First the geometrical acceptance is only of the order of $40 \%$ due to the presence of the window in the vacuum pipe and must be determined experimentally. Second, the average number of photons reaching simultaneously the detector at each beam crossing is larger than one (multi-photon regime). Hence the total energy per crossing is measured rather than the single photon spectrum. Third the small beam transverse dimension at LEP has to be taken into account in the single bremsstrahlung cross section evaluation [6]. In fact, at the LEP energies, there is a cut-off on the impact parameter integration due to the finite and small size of the beam.

The luminosity measurement is based on the relation:

$$
E_{\text {meas }}-E_{b c k g}=A L \int_{0}^{E_{\text {beam }}} \varepsilon(k) k \frac{d \sigma}{d k} d k
$$

where :

- $E_{\text {meas }}$ is the total measured energy in a given time interval;

- Ebckg is the background measured energy (the main contributions come from beamgas bremsstrahlung and inverse Compton scattered thermal photons $[3,7]$ );

- $\mathrm{L}$ is the integrated luminosity in the time interval;

- A is the geometrical acceptance

- $\mathrm{k}$ is the photon energy;

- Ebeam is the beam energy;

- $\mathrm{d} \sigma / \mathrm{dk}$ is the single bremsstrahlung differential cross section corrected for the finite beam size [6];

- $\varepsilon(k)$ is the photon energy detection efficiency.

The single beam radiation background $E_{\text {bckg }}$ is measured as a function of time when the separators are switched on in IP-1 (no collision condition); thus its value during collisions can be extrapolated and subtracted (see Sect.4).

The angular distribution of the single bremsstrahlung photons is expected to be a gaussian with a width related mainly to the beam divergence at the IP. By fitting the measured energy spatial distribution in the calorimeter modules one gets the acceptance $A$ and the beam transverse sizes [8]. 
Detector calibration and test carried out at the SPS (CERN) electron test beam $[2,5,9]$ provide the $\varepsilon(k)$ function.

In order to obtain a precise luminosity measurement one has to go through two main steps: (i) to fit the Ebckg behaviour before collisions and (ii), after the background subtraction, to fit the shape of the energy spatial distribution in the calorimeter modules [2,8]. At present this process is carried on off-line hence it is essentially a slow process. For quick monitoring one can already use the energy plot display (see Fig.s 2 and 3). Furthermore even the exact computation of luminosity could be implemented on-line with a more sophisticated program.

\section{The read-out system upgrade}

The 60 PMT outputs of the calorimeter are sent to four 16 channel charge integrating ADC modules (LeCroy 4300B/610 FERA). As the ADC conversion takes a maximum of $8.5 \mu \mathrm{sec}$ for an 11-bit sensitivity, data can be processed at the same rate of the LEP bunch crossing (occurring every $22 \mu \mathrm{sec}$ ) if the reading of the ADC buffer is carried out quickly enough. This suggests the use of the FERA ECL front port which has a $50 \mathrm{~ns} /$ word speed capability.

Before the upgrading, data were read into the computer ( $\mu$ VAX 3300) via straight CAMAC commands. The acquisition rate was limited to few hundred hertz. The system was certainly not optimized, but no amount of optimization would have permitted to reach the $22 \mu \mathrm{sec}$ transfer time, since the fastest CAMAC transmission time is about $1.5 \mu \mathrm{sec} /$ word.

Even this simple read-out was enough to perform a faster luminosity measurement than in the conventional luminometers at LEP [1] but the full capability of the method was not exploited. Therefore a different read-out scheme, requiring the use of a specially designed CAMAC module (FERAPROCESSOR) [10] to be coupled with each ADC, has been employed. The scheme is the following:

- the LEP beam instrumentation provides two signals: the bunch crossing signal (needed to synchronize the ADC gate) and the first bunch identifier signal (FBI);

- the ADC begins the conversion with the bunch crossing signal;

- the FERAPROCESSOR waits for the end of conversion (EOC) signal coming from the ADC;

- when the EOC signal is provided, it reads-out the ADC via its fast ECL front port;

- the bunch sequence is identified by using the FBI signal as a start of a 4-steps reading sequence; 
- the FERAPROCESSOR stores the 16 words read from the ADC in a corresponding memory ( 4 memories are used for the 4 bunches); subsequent data are added up to the content of this memory;

- the time needed to read-out the ADC and to update the memory is less than 11 $\mu \mathrm{sec}$ so that $\mathrm{ADC}$ conversion and data accumulation on the memory takes about 20 $\mu$ sec: all bunch crossings are processed and identified.

In the end the FERAPROCESSOR contains in its memory information about the energy integrated by each calorimeter module, separately for each of the 4 bunches. Data acquisition reaches its maximum intrinsic rate ${ }^{*}$.

The FERAPROCESSOR unit is a single slot CAMAC module and was designed, constructed and tested in Rome [10]. The main board elements are:

- a powerful microprocessor (DSP 56001 Motorola) with a 48 bit capability and a 75 nsec basic cycle; a $512 \times 24$ bit memory allows to load the acquisition program (PRGRAM); an aritmetic logic unit (ALU) and two banks of $256 \times 24$ bit of internal memory are used to store data (X,Y RAM) [11];

- a 32K x 24 bit high speed RAM bank (EXTRAM);

- a CAMAC bus interface;

- an ECL input front port;

- a RS232 serial communication interface.

The FERAPROCESSOR computing power is about 10 million of instructions per second (MIPS).

The program to read-out and process data is down loaded onto the PRGRAM and runned via standard CAMAC commands. On the FBI signal, the program jumps to an interrupt service routine. This routine waits for the EOC signal, then reads the 16word data from the ADC; old data in the X,Y RAM are moved in the ALU register and new data are added up to the old one; the result of the sum are moved again in the $\mathrm{X}, \mathrm{Y}$ RAM. This instruction sequence is repeated 4 times (i.e. separately for each bunch). Then the control is given again to the main program that waits for another FBI signal.

In this specific working scheme, where data from successive events are simply accumulated, the maximum number of events that can be processed depends only on the DSP and ADC word lengths, respectively 48 and 11 bits. So in our case it is $2^{37}=10^{11}$ events.

On a CAMAC reading request the program jumps to a CAMAC interrupt service routine that moves data from the X,Y RAM to the EXT RAM. Then control returns

\footnotetext{
* This is true at LEP in the four bunches scheme. In the eight bunches configuration the upper limit for the acquisition rate becomes $11 \mu \mathrm{sec}$. Neverthless this upper limit can be reached in a different readout scheme where data of a bunch are processed in parallel to the ADC conversion of the next bunch.
} 
to the DSP that continues data accumulation while data on the EXT RAM can be read via CAMAC and stored on the main computer ( $\mu$ VAX 3300).

\section{Test results}

A test has been performed during October-November 1991. Data have been taken mainly in separated beam parasitic conditions. Due to beam instabilities when colliding in IP-1, colliding beams have been available only twice: during fill-836 on October 23rd and during fill-866 on November 7 th.

During fill-836 collisions in IP- 1 started at a total current $I=2.1 \mathrm{~mA}$, after about 7 hours of beam coasting. Unfortunately after $40 \mathrm{sec}$ from crossing time the electron beam was lost due to a beam blow-up. In Fig. 2 the e.m. calorimeter signal ( $\mathrm{GeV} / \mathrm{crossing}$ ) as a function of time is shown for the 4 bunches in a period of 8 minutes. Each point corresponds to a $20 \mathrm{sec}$ measurement ( $2 \times 10^{5}$ crossings). The rise of the signal for all bunches is due to the start of the collisions, while the fall is due to the loss of the electron beam. The $30 \%$ difference between the single beam radiation level before and after the peak is interpreted as due to the different positron beam optics before and after the separator switching, so that the single beam radiation background to be subtracted is the one after the electron beam loss.

During fill-866 collisions have been provided at a total current of $1.8 \mathrm{~mA}(0.92$ $\mathrm{mA}$ positrons and $0.88 \mathrm{~mA}$ electrons) after about 4 hours of beam coasting. For about 10 minutes we have observed a stable colliding beams operation; then a beam blowup with a loss of $90 \%$ of the electron beam was observed. In Fig.3 the e.m. calorimeter signal is shown separately for the 4 bunches. The rise of the signal due to the start of the collisions, the 10 minutes long stable colliding beams operations and the drop due to the electron beam loss are again clearly seen.

In order to extract a luminosity value from the data collected during fill-866 one has to solve two problems: (i) single beam radiation background subtraction and (ii) acceptance evaluation.

(i) The single beam radiation level after the electron beam loss is evaluated by scaling down the radiation level before the collision by the $30 \%$ amount observed during the fill-836 (the level observed after the electron beam loss in fill-866 cannot be used due to about a $10 \%$ residual electron beam).

(ii) From the space distribution of the energy in the calorimeter modules one can infer that the photon beam during the 10 minutes collisions was centered out of the window (see Fig.4a) which makes the acceptance evaluation more uncertain. For practical reasons we were not able to correct the beam direction in such a short time. $A$ mean value $A \approx 10 \%$ can be obtained by fitting the distributions. In Fig. $4 \mathrm{~b}$ an 
example of a distribution measured in an old run (1990), where the acceptance was about $40 \%$, is shown for comparison [2].

Finally, using the average measured acceptance during the collision period, the luminosity can be evaluated from eq.(1) as shown in Fig.5. It is worth noting that each point corresponds to a $20 \mathrm{sec}$ measurement and has a $0.2 \%$ statistical uncertainty. In this case (fill 866), the systematic error is evaluated to be of the order of $10 \%$ and is almost entirely due to the acceptance evaluation.

\section{Conclusions}

The upgrade of the LEP-5 experiment has been successfully carried out: both the objectives of (a) exploiting the intrinsic high rate of the single bremsstrahlung method and (b) performing a separated bunch measurement are reached. In conclusion the possibility of performing very fast luminosity measurements at LEP by the single bremsstrahlung method has been demonstrated. The statistical uncertainty (less than $1 \%$ in few seconds) is negligible respect to the systematic one, being the latter of the order of 3-5\% with well centered beams.

This method would be very appealing in case a "spin rotator" magnet were installed in a LEP straight section in order to get longitudinal polarization. In fact in that case, as the detector should be placed only 40 meters away from the IP, the geometrical acceptance would be practically always $100 \%$.

\section{Acknowledgements}

We thank the group technician M.Bertino for the excellent work as well as the technical staff of the Officina elettronica - INFN Sezione di Roma. The collaboration of the LEP control room people is gratefully acknowledged. Finally we like to thank our colleague F.Garufi for helping us during the data taking. 


\section{References}

[1] R.Schmidt, Proceedings of the 3rd Workshop on LEP performance, Chamonix, France, 10-16 Jan 1993 - (CERN SL 93-19 DI)

[2] C.Bini et al., Nucl.Instr.\&Meth. A306 (1991) 467

[3] C.Bini et al, Phys.Lett. B262 (1991) 135

[4] Polarization at LEP, G.Alexander et al., Eds. CERN 88-06;

A.Blondel, CERN PPE/93-125

[5] M.Bertino et al, Nucl.Instr.\&Meth. A315 (1992) 327

[6] A.E.Blinov et al., Nucl.Instr.\&Meth.A273 (1988) 31 and references therein;

G.L.Kotkin et al., Phys.Lett. B221 (1989) 96

[7] A.Di Domenico, Particle Accelerator 39 (1992) 137

[8] C.Bini et al, CERN/LEPC 89-5 LEPC/I8 add.1;

C.Bini et al, CERN/LEPC 89-12 LEPC/I8 add. 2

[9] C.Bini et al. LEP-5 status report CERN/LEPC 90-01 M.91

[10] D.De Pedis, note in preparation

[11] Motorola technical note - DSP 56001 ADI 1290 Rev.1

\section{Figure captions}

Fig.1 - A sketch of LEP half straight section from IP-1 to the LEP-5 detector. Notice that photons leave the LEP vacuum pipe at about $300 \mathrm{~m}$ from IP-1.

Fig.2 - The electromagnetic calorimeter signal (GeV/crossing) as a function of time for the four bunches during fill-836.

Fig.3 - The electromagnetic calorimeter signal ( $\mathrm{GeV} / \mathrm{crossing}$ ) as a function of time for the four bunches during fill-866.

Fig.4 - (a) The space distribution of the energy in the calorimeter modules for the four bunches during collisions (fill-866 - November 1991) after background subtraction; (b) the space distribution of the energy in the calorimeter modules during collisions (fill-409 - August 1990) after background subtraction [2].

Fig.5 - Absolute luminosity per bunch $\mathrm{L}$ as a function of time for the four bunches during fill-866. 


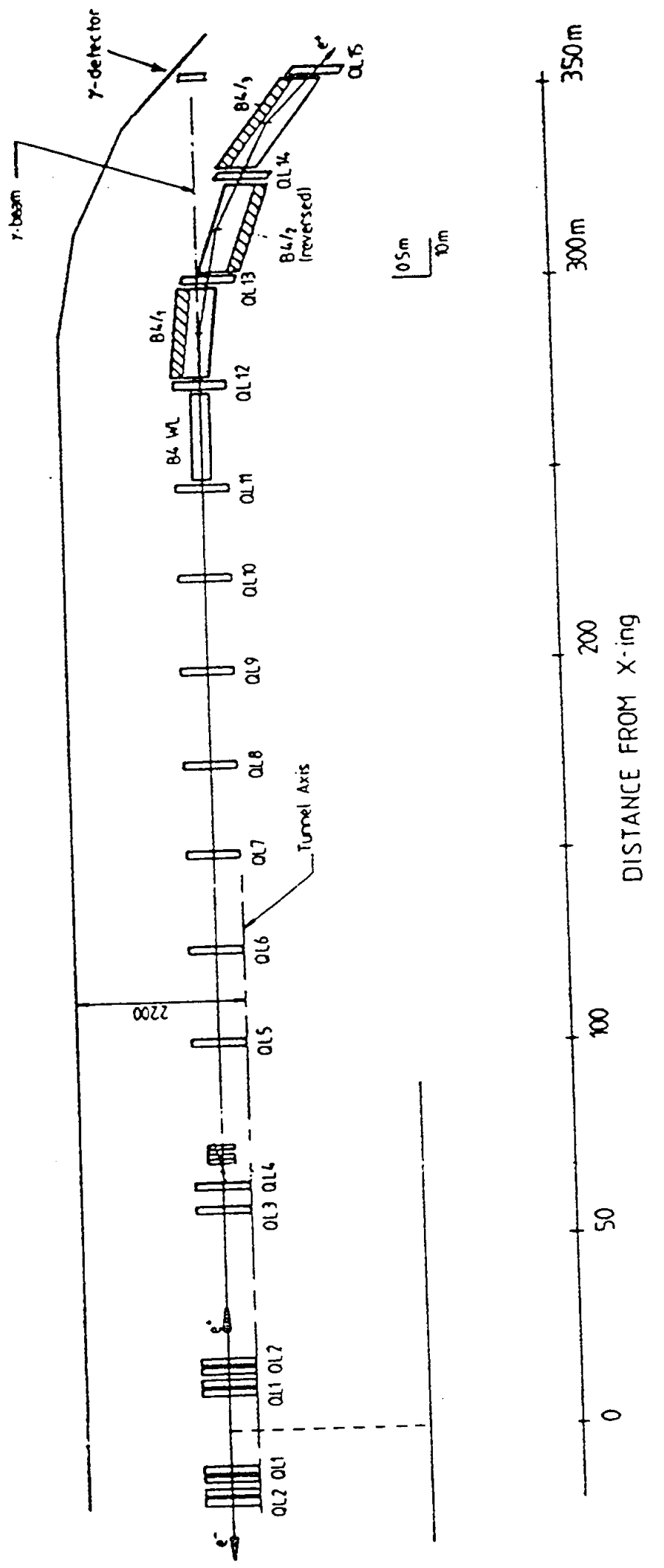



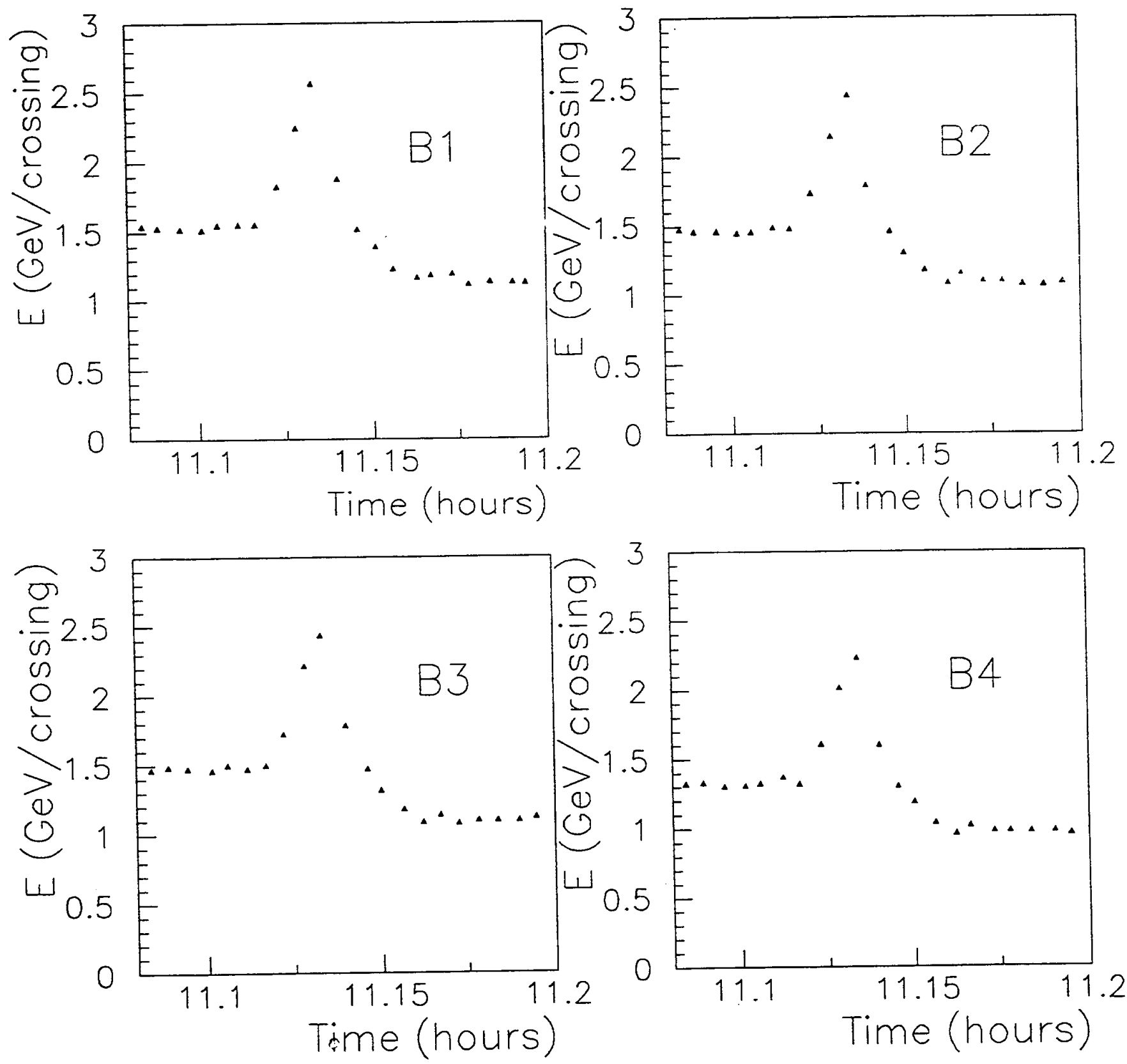

Fig.2 

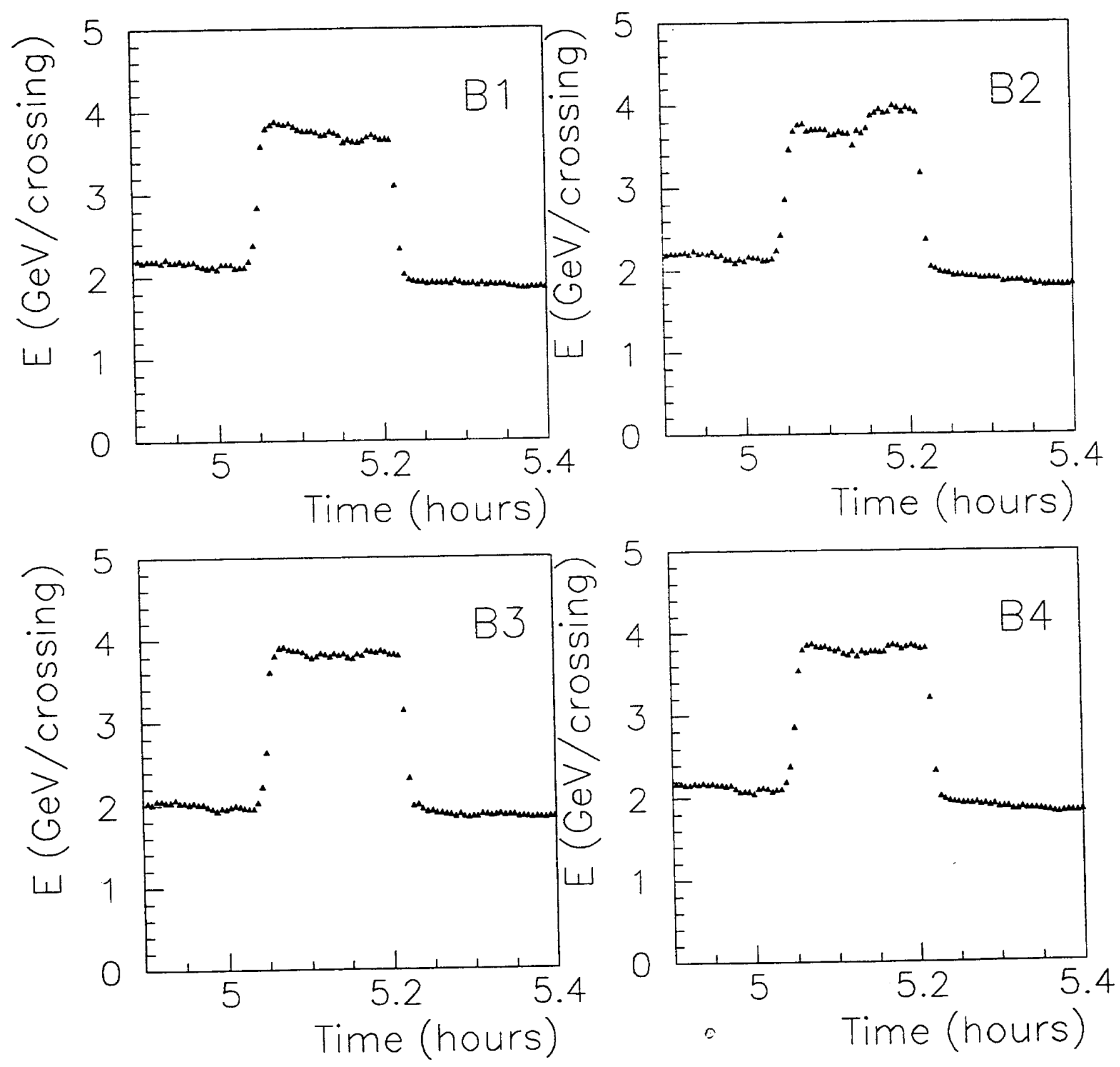

Fig.3 

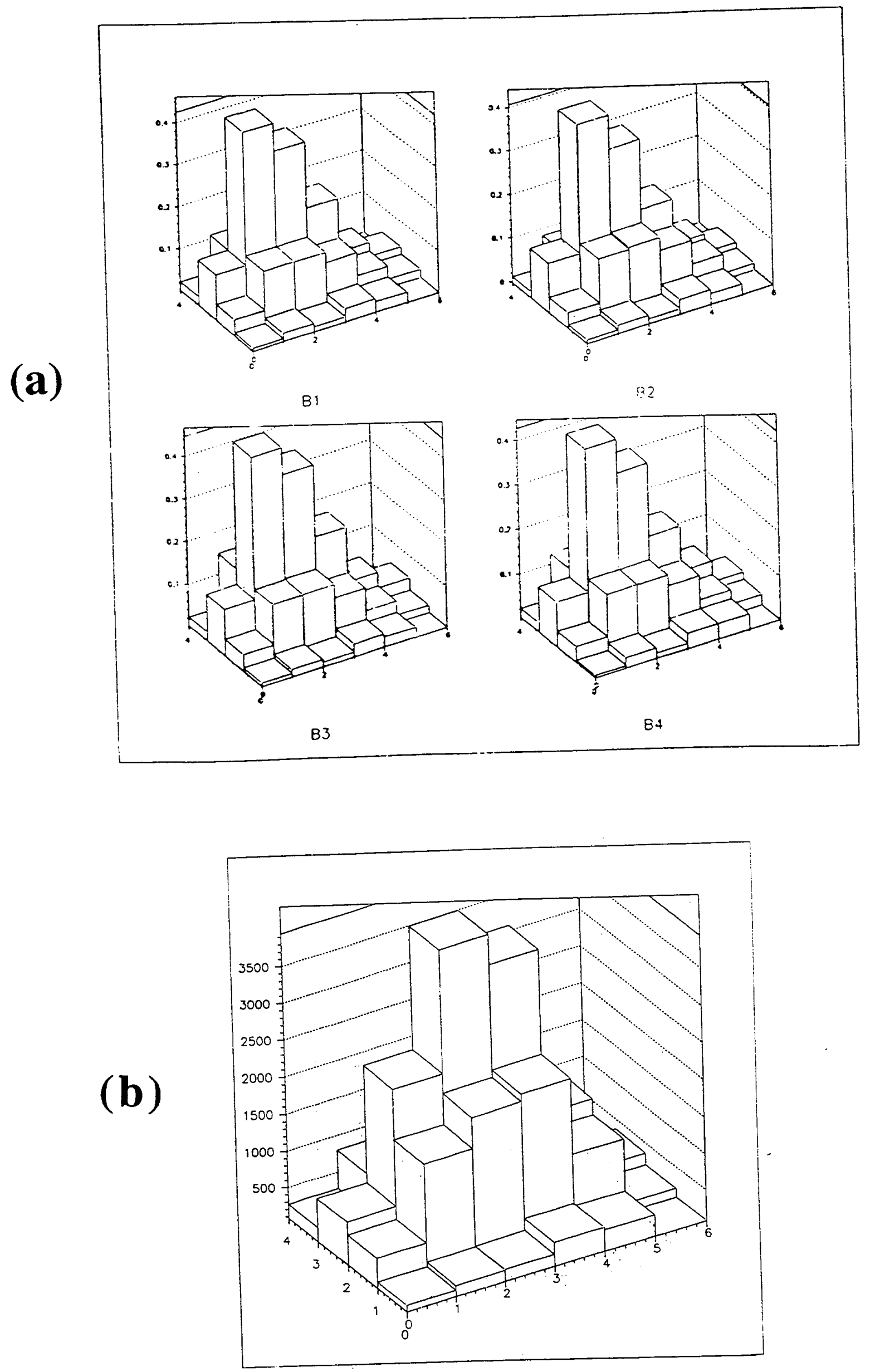

Fig.4 

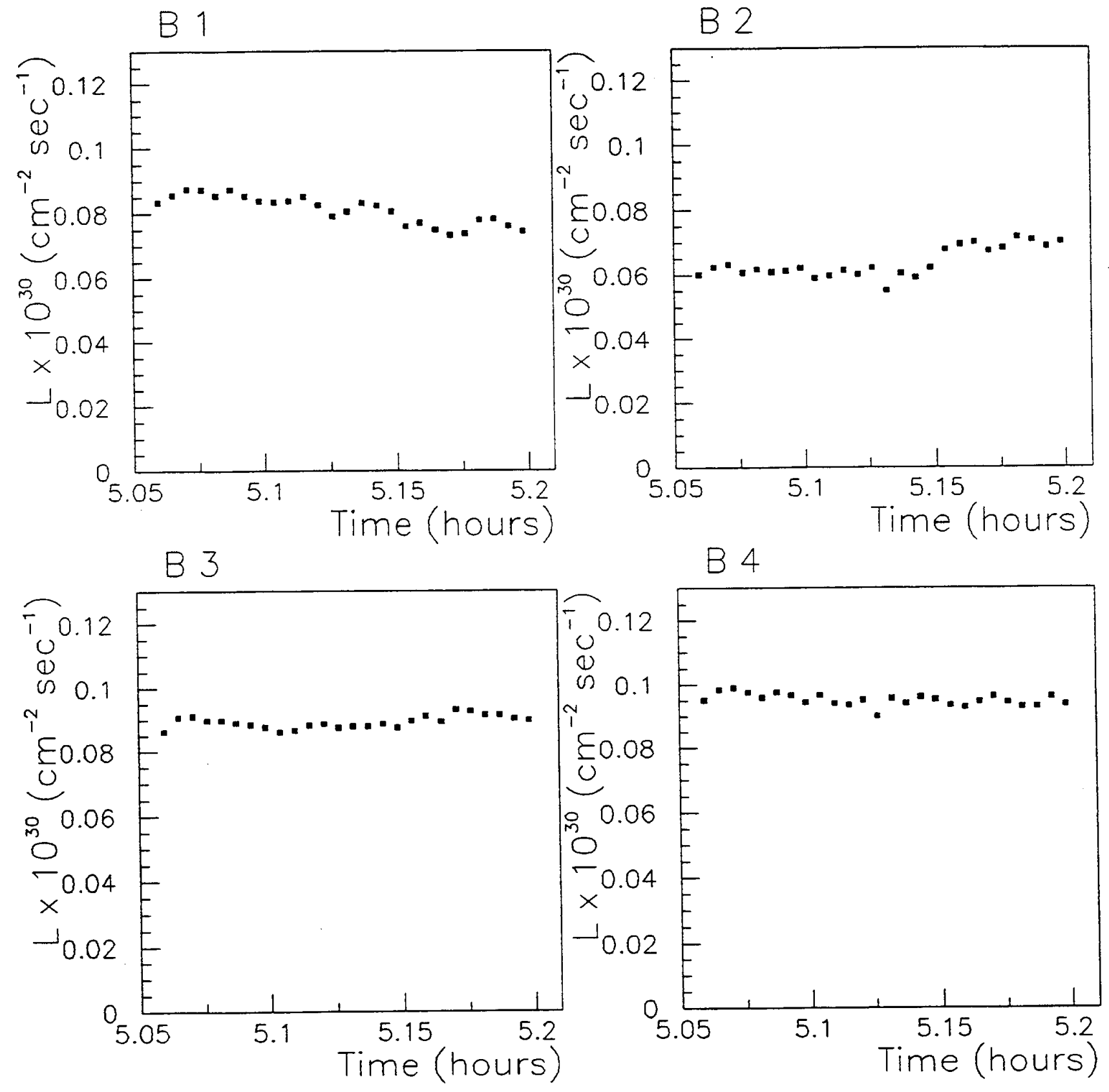

Fig.5 
